\title{
Fuzzy Integrals as a Tool for Obtaining an Indicator for Quality of Life.
}

\author{
Dick Botteldooren, Andy Verkeyn, Members, IEEE, Bernard De Baets, Peter Lercher
}

\begin{abstract}
Many governments and international organizations recognize the fact that classical economic indicators do not accurately reflect the quality of life in a country or region. Indicators that reflect the subjective evaluation of well-being or quality of life by inhabitants have to be constructed based on multiple criteria. Multi-criteria evaluation systems based on fuzzy integrals seem very well suited for this task as they tend to approximate overall quality assessment by inhabitants quite well. This paper discusses the choice of fuzzy integrals and analyses the suitability of the approach based on a survey with 2000 people.
\end{abstract}

\section{INTRODUCTION}

$\mathrm{W}$ HEN people are asked to assess their own state by questioning them about abstract concepts such as well-being or quality of life, a complicated cognitive process is started. Their fundamental judgment includes their enduring mood (e.g. happiness) as well as their evaluation of the self (e.g. satisfaction with one's physical and mental health and functioning) and its relation to the material and psychosocial environment (e.g. life satisfaction, work satisfaction) [1]. The conclusion an individual reaches for each of these individual aspects depends on the maximum and minimum conceivable satisfaction and thus is rather vague. The standards used depend on interactions with peers or one's own previous experience. After individual aspects are rated, the questioned person comes up with an aggregated answer to the global assessment question.

An increasing number of social scientists and philosophers believe that classical economic indicators for progress fall short because they fail to grasp the difference that different people and cultures attach to different things. A suggested alternative would be to measure progress as the change over time of average scores of happiness, which is roughly equivalent to terms such as "life satisfaction", "subjective quality of life", or "subjective wellbeing" [2]. As mentioned above, a lot of imprecision is introduced when questioning a population about such happiness indicators.

D. Botteldooren is with the Department of Information Technology of Ghent University, Gent Belgium (phone: +32 9264 9968, fax +329264 9969; e-mail: dick.botteldooren@intec.ugent.be).

A. Verkeyn was with the Department of Information Technology. He is now with Guidance Corporation, Brussels, Belgium (e-mail: verkeyn@guidance.be).

B. De Baets is with the Department of Applied Mathematics, Biometrics and Process Control, Ghent University, Gent, Belgium (email:

bernard.debaets@ugent.be)

P. Lercher is with the Department of Hygiene and Social Medicine, Medical University of Innsbruck, A-6020 Innsbruck, Austria (email: peter.lercher@i-med.ac.at)
One way to overcome this, is to construct the indicator from the answers to more basic questions that can more easily be grounded by the person questioned. This implies that at some point the answers to these questions will have to be aggregated to obtain an overall assessment. This is best done in line with the cognitive process a human observer would use for this purpose. In previous work [3], [4] we have successfully used fuzzy integrals to model the overall annoyance by noise and odor reported by people based on their response to individual sources of annoyance. Hence this paper proposes using fuzzy integrals for constructing an indicator for quality of life (QoL) or subjective well-being (SWB). In [5], Roubens studies the aggregation problem in general and summarizes the operators that fulfill the minimal properties of an aggregator: suitable limit conditions, monotonicity, compensativeness, and continuity. Choquet and Sugeno integrals are the more general operators in his overview and they reduce to the quasi-linear weighted average, weighted mean, weighted minimum and weighted maximum for special choices of the fuzzy measures involved. Thus considering the fuzzy integrals is a straight forward generalization of the simpler aggregation functions classically used for the application studied in this paper.

Section II discusses on a theoretical base which fuzzy integrals and fuzzy measures are suitable for weighted aggregation of different aspects of QoL. Section III looks at the outcome for a particular situation to evaluate if aggregation based on fuzzy integrals yields results that are logical, in particular in comparison to the answer to a question on life satisfaction.

\section{FUZZY INTEGRALS}

\section{A. Requirements imposed by the application at hand}

To achieve a high QoL, several conditions need to be fulfilled:

- primary and secondary needs should be easy to satisfy;

- the living environment should not impose too much stress on the organism;

- work (either housework or paid labor) should be performed in ideal conditions and at good working hours;

- illness or disabilities should be absent;

- the direct living environment should offer good social support;

- etc. 
Each of the higher level criteria mentioned above still suffer from a high degree of abstraction and are therefore not easily grounded by the interviewed. Thus each of them is further unraveled into more basic aspects. E.g. fulfillment of primary needs in today's society could be considered to be determent by the degree of satisfaction with shopping facilities, entertainment activities, schools and kindergarten, public transport, etc., in the community.

The two-stage aggregation of these criteria can be regarded as a multi-criteria decision making (MCDM) problem. Using a classical weighted linear average does not allow to accurately capturing some of the known effects:

1. Some of the criteria may be so strong that they dominate the aggregated value no matter what the degree of satisfaction of the other criteria. E.g. Extremely poor health may lead to poor QoL no matter how visually appealing the living environment is.

2. Cumulative exposure may lead to increased effect, or in MCDM language, the coalition between criteria is important.

3. Criteria may to some extend be complementary. This may be the case when satisfaction of one or the other criterion is sufficient for high QoL. More difficult to recognize is the situation where questions actually sample the same underlying aspect, e.g. mild traffic, no disturbing traffic noise, low perceived accident risk.

4. Many aspects of QoL are described by bipolar scales, e.g. ranging from "very unsatisfied" to "very satisfied".

5. Most questionnaire data is gathered using ordinal scales.

\section{B. Bipolar discrete Choquet integral with $k$-additive fuzzy measure}

We start this section by recalling the notion of fuzzy measures on which fuzzy integrals are defined.

Definition fuzzy measure [15]: A fuzzy measure $\mu$ on $X=$ $\left\{x_{1}, x_{2}, \ldots, x_{n}\right\}$ is a set function $\mu: X^{2} \rightarrow[0,1]$ satisfying:

- Boundary condition: $\mu(\varnothing)=0$.

- Normalization: $\mu(X)=1$

- Monotonicity: $\forall A, B \in X^{2}, A \subseteq B \Rightarrow \mu(A) \leq \mu(B)$.

This paper requires the properties of bipolar Choquet integrals, therefore we start by giving their definition.

Definition Symmetric Choquet integral [6]: Consider a fuzzy measure $\mu$ on $X$ and a mapping $f: X^{2} \rightarrow \mathfrak{R}$. The symmetric Choquet integral (also known as Šipoš integral) of $f$ w.r.t. $\mu$ is defined as

$$
\breve{C}_{\mu}(f)=C_{\mu}\left(f_{+}\right)-C_{\mu}\left(f_{-}\right)
$$

where $C_{\mu}$ is the classical Choquet integral [11],

$f_{+}=\left(f_{+}\left(x_{1}\right), f_{+}\left(x_{2}\right), \cdots, f_{+}\left(x_{n}\right)\right)$ and

$f_{-}=\left(f_{-}\left(x_{1}\right), f_{-}\left(x_{2}\right), \cdots, f_{-}\left(x_{n}\right)\right)$ with $f_{+}\left(x_{i}\right)=\max \left(f\left(x_{i}\right), 0\right)$ and $f_{-}\left(x_{i}\right)=\max \left(-f\left(x_{i}\right), 0\right)$ for all $x_{i} \in X$.

The symmetric Choquet integral allows symmetric processing of the positive and negative numbers. The zero is a "true" zero, and the underlying scale is a ratio scale.

A fuzzy measure $\mu$ on $X$ can also be represented as its Möbius transform $m: X^{2} \rightarrow \mathfrak{R}$ which is defined as

$$
m(A)=\sum_{B \subseteq A}(-1)^{|A-B|} \mu(B) .
$$

Definition $k$-additive fuzzy measure [8]: Consider $k \in\{1$, $2, \ldots, n\}$. A fuzzy measure $\mu$ on $X$ is called k-additive if its Möbius transform $m$ satisfies $m(A)=0$ whenever $|A|>k$ and there exists at least one subset $B$ of $X$ such that $|B|=k$ and $m(B) \neq 0$.

From [7] it is known that the Choquet integral can be expressed as a function of the Möbius transform $m$ of a fuzzy measure. Substituting this expression in the definition of the Sipos integral gives us,

$$
\begin{aligned}
\breve{C}_{\mu}(f) & =\sum_{A \subseteq X} m(A)\left[\min _{i \in A} f^{+}\left(x_{i}\right)-\min _{i \in A} f^{-}\left(x_{i}\right)\right\rfloor \\
& =\sum_{A \subseteq X^{+}} m(A) \min _{i \in A} f\left(x_{i}\right)+\sum_{A \subseteq X^{-}} m(A) \max _{i \in A} f\left(x_{i}\right)
\end{aligned}
$$

where $X^{+}=\left\{x_{i} \mid f\left(x_{i}\right) \geq 0\right\}$ and $X^{-}=X-X^{+}$.

Following Grabisch [9] we can also write the Choquet integral with a 2-additive fuzzy measure as a function of Shapley value $v_{i}$ and interaction index $I_{i j}$ because these are easier to interpret and to generate by the field expert that has to provide the weights. The Shapley value is given by

$$
v_{i}=\sum_{K \subset N-\{i\}} \frac{k !(n-k-1) !}{n !}[\mu(K \cup\{i\})-\mu(K)]
$$

with $\mathrm{k}:=|\mathrm{K}|$ and can be interpreted as the weights in a linear weighted sum. The interaction index $I_{i j}$ describes the interaction between $i^{\text {th }}$ and $\mathrm{j}^{\text {th }}$ criterion and is defined as:

$$
\begin{aligned}
I_{i j} & =\sum_{K \subset N-\{i, j\}} \frac{k !(n-k-2) !}{(n-1) !} \\
& {[\mu(K \cup\{i, j\})-\mu(K \cup\{i\})-\mu(K \cup\{j\})+\mu(K)] }
\end{aligned}
$$

The 2-additive Choquet integral is written as:

$$
\begin{aligned}
C_{\mu}(f)=\sum_{I_{i j}>0}\left(f\left(x_{i}\right) \wedge f\left(x_{j}\right)\right) I_{i j} & +\sum_{I_{i j}<0}\left(f\left(x_{i}\right) \vee f\left(x_{j}\right)\right)\left|I_{i j}\right| \\
& +\sum_{i=1}^{n} f\left(x_{i}\right)\left(v_{i}-\frac{1}{2} \sum_{j \neq i}\left|I_{i j}\right|\right)
\end{aligned}
$$

The first sum explicitly expresses the additional effect of fulfilling two criteria, $x_{i}$ and $x_{j}$, together. The second sum in this expression allows implementing the situation where fulfilling one of the two criteria is sufficient.

The bipolar Choquet integral with k-additive fuzzy measures provides a solution for the requirements 2-4 mentioned above. It is not adequate to solve issue 1 and is not particularly suitable with regard to issue 5 . 


\section{Bipolar discrete Sugeno integral with k-maxitive fuzzy measure}

Let's start by recalling the definition of the symmetric Sugeno integral.

Definition Symmetric Sugeno integral [13]: Consider a fuzzy measure $\mu$ on $X$ and a mapping $f: X \rightarrow[-1,1]$. The symmetric Sugeno integral of $f$ w.r.t. $\mu$ is defined as

$$
\breve{S}_{\mu}(f)=S_{\mu}\left(f_{+}\right) \oplus\left(-S_{\mu}\left(f_{-}\right)\right)
$$

where where $\mathrm{S}_{\mu}$ is the classical Sugeno integral [15],

$f_{+}=\left(f_{+}\left(x_{1}\right), f_{+}\left(x_{2}\right), \cdots, f_{+}\left(x_{n}\right)\right)$ and

$f_{-}=\left(f_{-}\left(x_{1}\right), f_{-}\left(x_{2}\right), \cdots, f_{-}\left(x_{n}\right)\right)$ with

$f_{+}\left(x_{i}\right)=\max \left(f\left(x_{i}\right), 0\right)$ and $f_{-}\left(x_{i}\right)=\max \left(-f\left(x_{i}\right), 0\right)$ for all

$x_{i} \in X$, and the symmetric maximum is defined as $a \oplus b=$

$\operatorname{sign}(a+b)(\max (|a|,|b|)$. Note that $f$ should be a function on a symmetric ordinal scale in $[-1,1]$.

Similar to the notions of a Möbius transform and kadditive fuzzy measures, the concepts possibilistic Möbius transform and k-maxitive fuzzy measures have been defined.

A fuzzy measure $\mu$ on $X$ can be represented by its possibilistic Möbius transform $m^{\vee}=X^{2} \rightarrow[0,1]$ which is defined as [10]

$$
m^{\vee}(A)=\left\{\begin{array}{cc}
\mu(A) & \left(\mu(A)>\max _{B \subset A} \mu(B)\right) \\
0 & (\text { otherwise })
\end{array}\right.
$$

Definition k-maxitive fuzzy measure [10]: Consider $k \in$ $\{1,2, \ldots, n\}$. A fuzzy measure $\mu$ on $X$ is called k-maxitive if its possibilistic Möbius transform $m^{\vee}$ satisfies $m^{\vee}(A)=0$ whenever $|A|>k$ and there exists at least one subset $B$ of $X$ such that $|B|=k$ and $m^{\vee}(B) \neq 0$.

The classical Sugeno integral of $f: X \rightarrow[0,1]$ w.r.t. a kmaxitive fuzzy measure $\mu$ can be expressed as a function of the possibilistic Möbius transform $m^{\vee}$ of $\mu$ [12],

$$
S_{\mu}(f)=\max _{A \subseteq X} \min \left(m^{\vee}(A), \min _{i \in A} f\left(x_{i}\right)\right)
$$

The weights in this expression are to be interpreted as limiting the extent of the impact of a particular criterion, or combination of criteria on the aggregated result.

The Sugeno integral works on ordinal scales which is - at least theoretically - a benefit if the questionnaire uses such scales. The Sugeno integral not being strictly increasing and not satisfying weak separability is a disadvantage in decision making [14].

Thus Sugeno with k-maxitive clearly satisfy requirements 1, 4 and 5. Theoretically requirements 2 and 3 are also fulfilled but since discriminating fine detail is rather difficult using Sugeno integrals, practical implementations do not easily show the effect.

\section{Ordinal to cardinal scale translation}

To use the Choquet integral in practice, ordinal scales used in questionnaires must be translated to real numbers (in the interval $[0,1])$. It has been shown for the particular case of a question with regard to the intensity of noise annoyance that those questioned have a notion of where the labels used to identify an ordinal scale should be positioned on a continuous axis [16], [17]. The meaning of the labels themselves is best represented by a fuzzy set to express its imprecision. In the context of the problem studied in this paper, using fuzzy sets to represent labels used in questionnaires may complicate the process too much in case a Choquet integral is chosen for aggregation. Thus it was decided to represent the labels by single crisp numbers, not necessarily distributed equidistantly over the available universe.

The Sugeno integral with k-maxitive measure does not suffer from the ordinal scale problem. However, if the different aspects involved in constructing the indicator for QoL sample very different dimensions of life using very different scales to represent the answer, one still has to take critical decisions on the order relationship. E.g. is good health higher or lower than high quality shopping facilities in the area when integrating for QoL?

\section{E. Hierarchically structured decision making}

In Subsection A, it was discussed how the construct of a concept such as quality of life or subjective well-being could be unraveled into its fundamental components. This naturally leads to a multi-level or hierarchical aggregation process. Thus it makes sense to use at least a two-step aggregation operator as well. The main advantage of this approach is that the field expert can more easily assign weights when the problem is stated in a logical hierarchical way. It can nevertheless be proven that using Choquet and Sugeno integrals combined with k-additive or k-maxitive fuzzy measures in a two step aggregation process do not introduce any new features [19]. More exotic measures however allow changing between types of integral by using a two step procedure [18].

\section{APPLICATION TO THE BRENNER PASS AREA}

\section{A. Description of the survey}

A phone survey was conducted with 2002 inhabitants of the Brenner pass area. This Alpine region extends between Austria and Italy and consists of many small villages, some of which form a very rural community and are rather remote. This diversity makes this region well suited for this test. The survey was announced as an investigation into the personal appreciation of life, living environment, and health. It contained several questions (Table I) that can be used to assess quality of life of the respondents. The hierarchical structure of the construction of a QoL indicator based on the answers to these questions is shown in Fig. 1. 


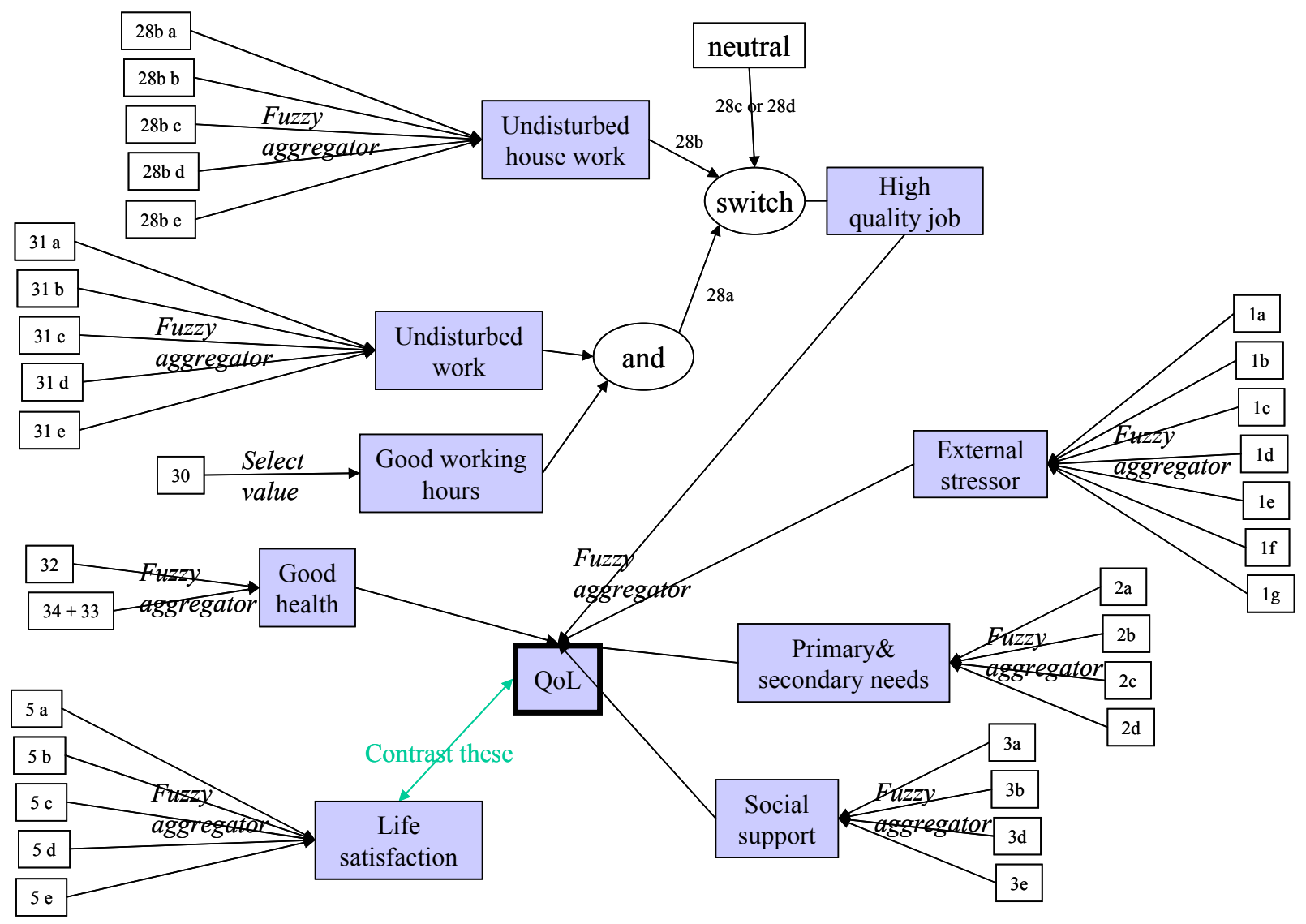

Fig. 1. Hierarchical structure of the construct of a QoL indicator.

\section{B. Weights}

The key topic in this and the following sections is the choice of the best possible aggregation scheme based on fuzzy integrals. In the written paper, two aggregation schemes are compared: the symmetric Sugeno integral with a 1-maxitive measure and the symmetric Choquet integral (Sipos) with a 1-additive measure. Note that in these cases, the integrals reduce to much simpler aggregators. The meaning of the singleton fuzzy measure - briefly called weight $w_{i}-$ is different in both schemes. In the 1-maxitive Sugeno integral, the weights determine the maximum extend to which an answer can influence the aggregated result. All of the answers from the survey that are included in the QoL indicator use an ordinal scale. One could easily base a Sugeno integral construction purely on the order of labels used in these scales. For simplicity, we nevertheless opted for translating the labels to crisp equidistant points in the interval $[0,1]$ or $[-1,1]$ if the interval is bipolar. The weights then must be within the interval $[0,1]$ and at least one of the weights must equal 1 in each aggregation. These weights are determined by field experts (Table I). Note that this modus operandus implies that the outcome of the aggregation can differ from a value corresponding to one of the labels used on the ordinal scale.
TABLE I

WEIGHTS USED IN THE AGGREGATIONS BASED ON 1-MAXIMATIVE SUGENO INTEGRAL

\begin{tabular}{|c|c|c|c|c|c|c|c|}
\hline & \multicolumn{2}{|c|}{ stressors } & \multicolumn{2}{|c|}{ needs } & social & job & health \\
\hline QoL & & .6 & & & 0.8 & 0.8 & 1 \\
\hline Q1 & air & noise & odor & waste & landscape & traffic & industry \\
\hline stressors & 1 & 1 & 1 & 0.8 & 0.5 & 0.8 & 0.8 \\
\hline Q2 & sho & ping & recre & tion & schools & public $\mathrm{t}$ & ransport \\
\hline needs & & 8 & 0 . & & 0.5 & 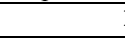 & 1 \\
\hline Q3 & appear & nce & uality o & living & security & neighbour & ly support \\
\hline social & 0 . & & 0. & & 0.8 & 1 & \\
\hline $\bar{Q} 28 \mathrm{~b} \&$ & \& Q31 & noise & odc & & eath/cold & vibrations & dirt \\
\hline $\begin{array}{l}\text { stressc } \\
\text { workp }\end{array}$ & $\begin{array}{l}\text { ors at } \\
\text { place }\end{array}$ & 1 & 0 . & & 1 & 0.8 & 0.5 \\
\hline & & & & & 232 & Q & 34 \\
\hline & healt & & & & 1 & 1 & \\
\hline
\end{tabular}

The weights in the 1-additive Choquet integral have the meaning of real weights in the multiplicative sense. The same mapping of the ordinal scales from the questionnaire to the continuous intervals $[0,1]$ or $[-1,1]$ is used, but now the result is interpreted as a cardinal scale. For maximal comparability between both approaches, the weights of Table I are used, but a normalization is applied to guarantee that the outcome of the aggregation is within the interval $[-1,1]$. 
TABLE II

QUESTIONS USED IN THE BRENNER PASS QUESTIONNAIRE

\begin{tabular}{|c|c|}
\hline nr. & Question \\
\hline 32 & When you think about the past 12 months, how would you assess your general health status? (very good, good, acceptable, less good, bad) \\
\hline $28 \mathrm{~b}$ & --- by Vibrations? (never, seldom, sometimes, mostly, always) \\
\hline $28 \mathrm{c}$ & --- by Odour/exhaust fumes? (never, seldom, sometimes, mostly, always) \\
\hline $28 \mathrm{~d}$ & --- by Dust/dirt? (never, seldom, sometimes, mostly, always) \\
\hline $31 \mathrm{~b}$ & --- by Vibrations? (never, seldom, sometimes, mostly, always) \\
\hline $31 \mathrm{c}$ & --- by Odor/exhaust fumes? (never, seldom, sometimes, mostly, always) \\
\hline $31 \mathrm{~d}$ & --- by Dust/dirt? (never, seldom, sometimes, mostly, always) \\
\hline $31 \mathrm{e}$ & --- by Heath/cold/dampness? (never, seldom, sometimes, mostly, always) \\
\hline 30 & Do you work: 2 shifts during the day / also during the night (22h-06h)/ on Saturday or Sunday / normal working hours during the day \\
\hline 1a & Please think about your community - Do you have reasons to complain about air pollution? (not at all, lesser, sizeable, a great many) \\
\hline $1 \mathrm{~b}$ & --- about noise? (not at all, lesser, sizeable, a great many) \\
\hline $1 \mathrm{~g}$ & --- about industry and small enterprises in your neighborhood? (not at all, lesser, sizeable, a great many) \\
\hline $2 \mathrm{a}$ & $\begin{array}{l}\text { Please think about your community - How satisfied are you with shopping possibilities? (very satisfied, quite satisfied, neither satisfied nor } \\
\text { unsatisfied, rather unsatisfied, very unsatisfied) }\end{array}$ \\
\hline $2 b$ & --- with variety of (recreational) activities? (very satisfied, quite satisfied, neither satisfied nor unsatisfied, rather unsatisfied, very unsatisfied) \\
\hline $2 \mathrm{c}$ & --- with kindergarten and schools? (very satisfied, quite satisfied, neither satisfied nor unsatisfied, rather unsatisfied, very unsatisfied) \\
\hline $2 \mathrm{~d}$ & --- with public transport? (very satisfied, quite satisfied, neither satisfied nor unsatisfied, rather unsatisfied, very unsatisfied) \\
\hline $3 \mathrm{a}$ & $\begin{array}{l}\text { Please think about the direct neighborhood }(200 \mathrm{~m}) \text { of your house - How satisfied are you with the appearance / the attractiveness of the } \\
\text { neighborhood? (very satisfied, quite satisfied, neither satisfied nor unsatisfied, rather unsatisfied, very unsatisfied) }\end{array}$ \\
\hline $3 b$ & --- with the quality of living in the neighborhood? (very satisfied, quite satisfied, neither satisfied nor unsatisfied, rather unsatisfied, very unsatisfied) \\
\hline $3 \mathrm{~d}$ & --- with the general safety of the neighborhood? (very satisfied, quite satisfied, neither satisfied nor unsatisfied, rather unsatisfied, very unsatisfied) \\
\hline $3 \mathrm{e}$ & $\begin{array}{l}\text {--- with the neighborly support in the neighborhood? (very satisfied, quite satisfied, neither satisfied nor unsatisfied, rather unsatisfied, very } \\
\text { unsatisfied) }\end{array}$ \\
\hline
\end{tabular}

\section{Comparison between QoL indicator and life satisfaction}

On top of the questions that are use to construct the QoL indicator, the survey also contains a set of questions related to general life satisfaction (Table I, Q5). Subjective evaluation of quality of life and general life satisfaction are not necessarily the same, but it makes sense to expect that both quantities are related. The QoL indicator will be used to evaluate the progress of groups of people, hence its quality can be checked on the basis of group-averaged values rather than individual assessments. To check whether the QoL indicator based on fuzzy integral aggregation gives expectable results, community averaged QoL and life satisfaction are compared in Fig. 2. A linear regression through these values gives a Pearson's $r^{2}$ equal to 0.65 if the 1-maxitive Sugeno aggregation and 0.54 for the 1-additive Choquet aggregation.

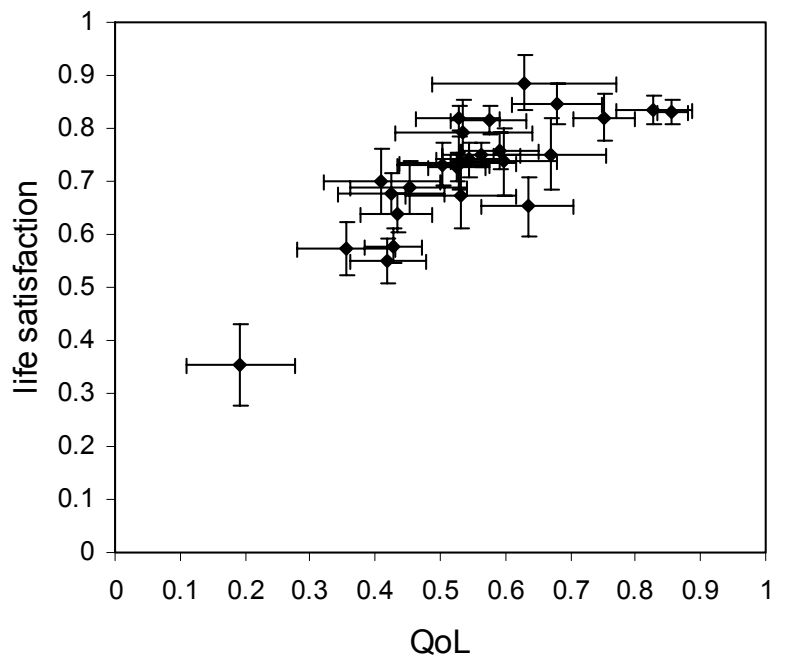

a) 


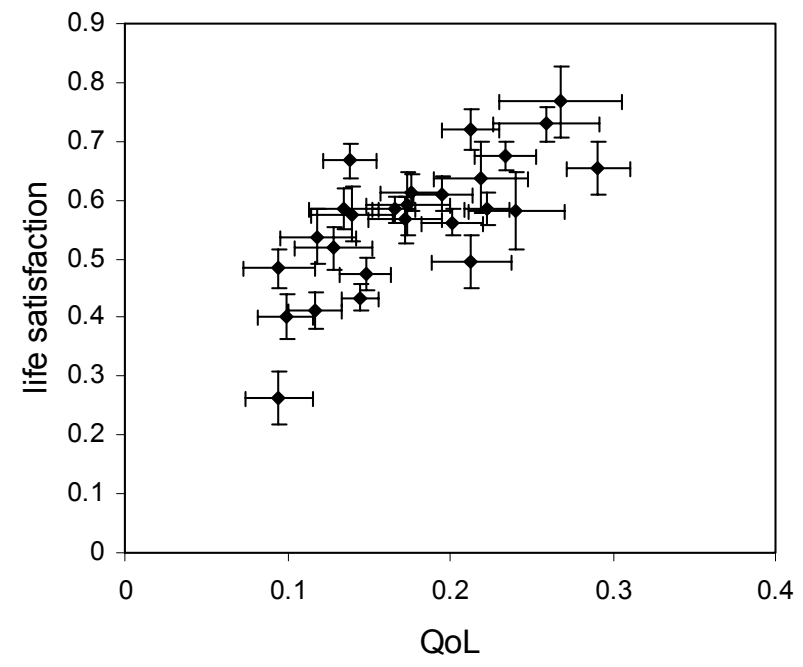

Fig. 2. Mean value and standard-error for Life satisfaction versus QoL indicator per community: a) 1-maximative Sugeno; b) 1-additive Choquet.

\section{QoL indicator as a function of dependent variables}

By investigating the constructed QoL indicator as a function of one of the variables used to construct it, leaving the other variables to float over the population under study, a clear picture is obtained of the importance of this variable in QoL, including possible correlations to other quantities. As an example, Fig. 3 shows the QoL as a function of noise annoyance (Q1b). The saturating impact of a noisy living environment on QoL as noise annoyance increases is clear if the 1-maxitive Sugeno integral is used for aggregation. Using a 1-additive Choquet integral results in an almost linear dependence. The result shown in Fig. 3a corresponds best with what one would expect for this variable.

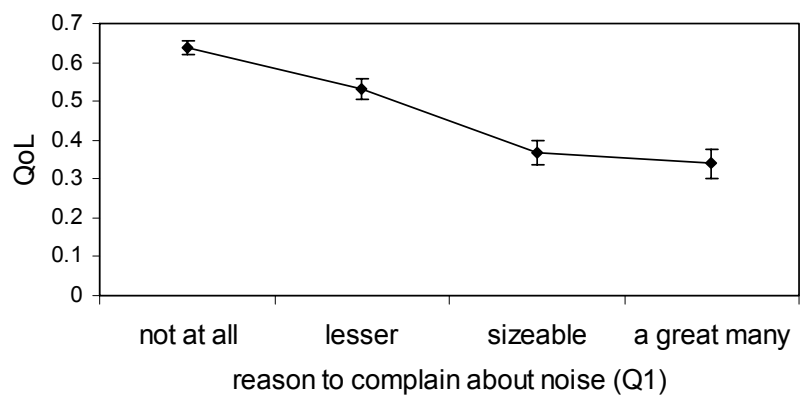

a)

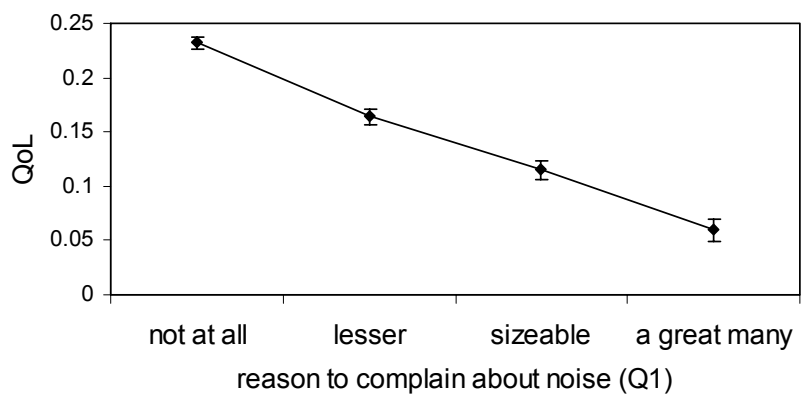

b)

Fig. 3. Aggregated QoL as a function of one of the variables in the construct: noise annoyance: a) 1-maxitive Sugeno; b) 1-additive Choquet.

\section{E. QoL indicator as a function of independent variables}

Studying the behavior of the constructed QoL as a function of an independent variable may shed more light on the suitability of either of the approaches used. A natural choice consists in looking at the effect of age (Fig. 4). It is observed that life satisfaction is rated somewhat lower for people around the age of 45-54. The QoL indicator that is constructed using the hierarchical model in Fig. 1, shows the same trend for both aggregators studied. There is nevertheless an observable difference. If a 1-maxitive Sugeno aggregation is used, the ratio of life satisfaction to calculated QoL increases, thus older people are more satisfied for the same QoL. If a 1-additive Choquet aggregation is used, the trend is precisely opposite. We could find no evidence in literature that allowed us to conclude which of these trends corresponds better to reality.

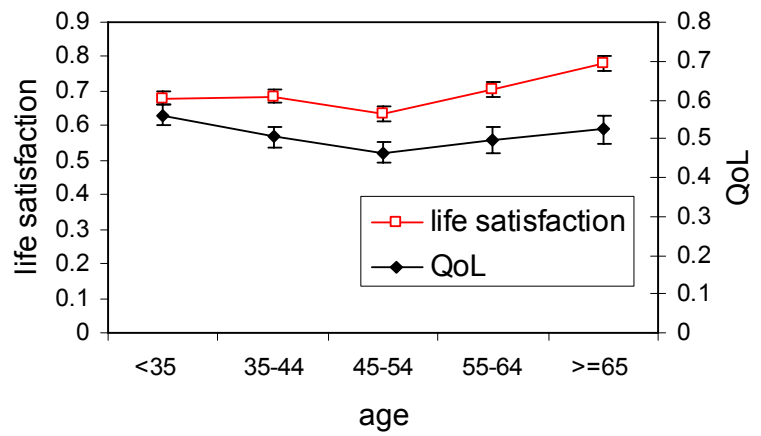

a)

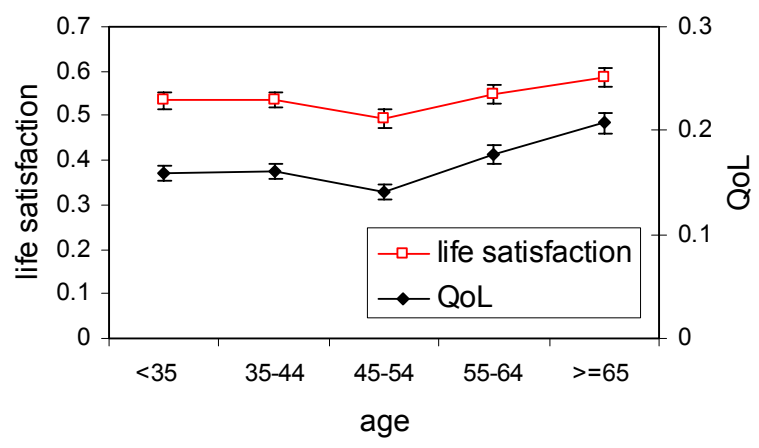

b)

Fig. 4. Life satisfaction and $\mathrm{QoL}$ as a function of an independent variable: age: a) 1-maxitive Sugeno; b) 1-additive Choquet.

\section{CONCLUSION}

Fuzzy integrals seem useful for implementing the aggregation processes that are needed to construct an indicator for quality of life or subjective well being. On a theoretical basis we have shown that these integrals, if combined with a suitable fuzzy measure, can meet most of the requirements for such aggregation. A test on field data involving 2000 respondents indirectly showed that the obtained results make sense.

When comparing Sugeno and Choquet integrals for this application theoretically, both seem to have several advantages and disadvantages. In particular the saturating behavior of Sugeno integrals is appreciated while the weak 
separability of the Choquet integral is appreciated. Comparison of a 1-additive Choquet aggregation to a 1maxitive Sugeno aggregation on field data results in a light subjective preference for the 1-maxitive Sugeno implementation.

\section{REFERENCES}

[1] J. Siegrist, "Subjective well-being: new conceptual and methodological developments in health-related social sciences," ESF SCSS Exploratory Workshop on 'Income, Interactions and Subjective Well-Being', Paris, 25-26 September 2003

[2] F. Heylighen and J. Bernheim, "Measuring global progress through subjective well-being," in: Proceedings of the III Conference of the ISQOLS, 2001

[3] A. Verkeyn, D. Botteldooren, B. De Baets, G. De Tre, "Sugeno integrals for the modelling of noise annoyance aggregation", Lecture notes on artificial inteligence 2715, Springer-Verlag, 2003, 277-284

[4] A. Verkeyn, D. Botteldooren, B. De Baets, G. De Tre, Modeling annoyance aggregation with Choquet integrals, Proceedings of Eurofuse Workshop on Information Systems, Villa Monastero, Varenna, Italy, Sep. 2002, pp. 259-264

[5] M. Roubens, "Fuzzy sets and decision analyses," Fuzzy Sets and Systems 90, pp. 199-206, 1997

[6] M. Grabisch and C. Labreuche, "Fuzzy measures and integrals in MCDA," in Multiple Criteria Decision Analysis, J. Figueira, S. Greco, and M. Ehrgott, Eds. Kluwer Academic Publishers, 2004.

[7] A. Chateauneuf and J. Y. Jaffray, "Some characterizations of lower probabilities and other monotone capacities through the use of Möbius inversion," Math. Social. Sciences, vol. 17, pp. 263-283, 1989.

[8] M. Grabisch, "k-order additive discrete fuzzy measures and their representation," Fuzzy sets and systems, vol. 92, pp. 167-189, 1997.

[9] M. Grabisch, "Alternative representations of discrete fuzzy measures for decision making," International Journal of Uncertainty, Fuzziness and Knowledge-Based Systems, vol. 5, pp. 587-607, 1997.

[10] R. Mesiar, "Generalizations of k-order additive discrete fuzzy measures," Fuzzy sets and systems, vol. 102, pp. 423-428, 1999.

[11] G. Choquet, "Theory of capacities," Annales de l'Institut Fourier, vol. 5, pp. 131-295, 1953.

[12] J.-L. Marichal, "Aggregation operators for multicriteria decision aid," Ph.D. dissertation, University of Li'ege, Belgium, 1998.

[13] M. Grabisch, "The symmetric Sugeno integral," Fuzzy sets and systems, vol. 139, pp. 473-490, 2003.

[14] M. Grabisch and C. Labreuche, "Fuzzy measures and integrals in MCDA," in Multiple Criteria Decision Analysis: State of the Art Surveys, Series: International Series in Operations Research \& Management Science, Vol. 78, J. Figueira; S. Greco; M. Ehrgott, Eds., Springer, 2005

[15] M. Sugeno, "Theory of fuzzy integrals and its applications," Ph.D. dissertation, Tokyo Institute of Technology, Japan, 1974

[16] D. Botteldooren, A. Verkeyn, C. Cornelis, M. De Cock, "On the Meaning of Noise Annoyance Modifiers: A Fuzzy Set Theoretical Approach", Acta Acustica united with Acustica 88 (2), March/April 2002, pp. 239

[17] A. Verkeyn, M. De Cock, D. Botteldooren, E. E. Kerre, "Generating Membership Functions for a Noise Annoyance Model from Experimental Data," In: Studies in Fuzziness and Soft computing: Soft computing in measurement and information acquisition (Vol. 127), L. Reznik, V. Kreinovich (eds), Springer-Verlag, 2003

[18] Y. Narukawa and V. Torra, "Twofold integral and multi-step Choquet integral," KYBERNETIKA 40 (1): pp. 39-50 2004

[19] R. Mesiar and D. Vivona, "Two-step integral with respect to fuzzy measure," Tatra Mt. Math. Publ. 16, pp. 359-368, 1999 


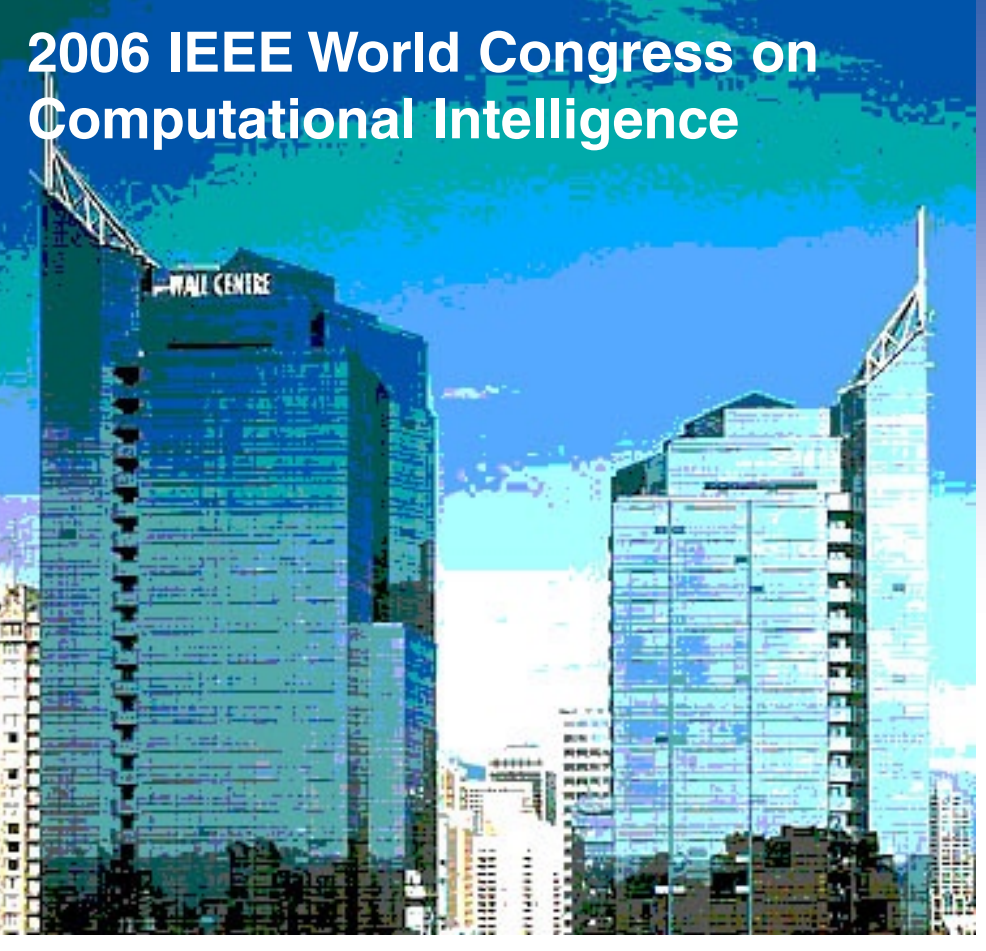

\section{A Joint Conference of the:}

2006 International Joint Conference on Neural Networks 2006 IEEE International Conference on Fuzzy Systems 2006 IEEE Congress on Evolutionary Computation

Conference Information

Program at a Glance

Technical Program

Author Index

Area Maps and Floor Maps

Sponsors:

- IEEE
IEEE

Computation
Intelifigence Intelligen
Society

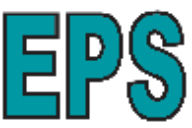

Evolutionary Programming Society
The

Internationa

Neural

Society

\section{Call for Papers}

\section{(๑) 2006}

Help

\section{Co-Sponsors:}

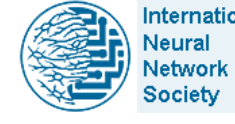

